\title{
Human Exposure to Hantaviruses Associated with Rodents of the Murinae Subfamily, Madagascar
}

\author{
Harinirina Aina Rabemananjara, ${ }^{1}$ Vololoniaina Raharinosy, ${ }^{1}$ Ravo Michèle Razafimahefa, \\ Jean Pierre Ravalohery, Jean Théophile Rafisandratantsoa, Soa Fy Andriamandimby, \\ Minoarisoa Rajerison, Soanandrasana Rahelinirina, Aina Harimanana, Judickaelle Irinantenaina, \\ Marie-Marie Olive, Christophe Rogier, Noël Tordo, Rainer G. Ulrich, Jean-Marc Reynes, \\ Stéphane Petres, Jean-Michel Heraud, ${ }^{2}$ Sandra Telfer, ${ }^{2}$ Claudia Filippone ${ }^{2}$
}

\begin{abstract}
We conducted a national human serologic study of a hantavirus detected in Madagascar rodents using a commercial kit and a new ELISA targeting the virus. Our results suggest a conservative estimate of $2.7 \%(46 / 1,680)$ lgG seroprevalence. A second single-district study using the new ELISA revealed a higher prevalence $(7.2 \% ; 10 / 139)$.
\end{abstract}

$\mathrm{H}$ antaviruses belonging to the genus Orthohantavirus, family Hantaviridae, are frequently zoonotic. Rodents are the usual reservoirs of human pathogenic hantaviruses and typically do not show obvious signs of disease $(1,2)$. Transmission to humans usually occurs by inhalation of aerosols contaminated with urine or feces of infected reservoir animals (3). Hantaviruses are responsible for the severe illness hemorrhagic fever with renal syndrome (HFRS) and a milder form, nephropathia epidemica (NE), as well as for hantavirus cardiopulmonary syndrome (HCPS) (1).

Recent studies have described the geographic distribution and host range of novel hantaviruses in Africa and the Indian Ocean (4-6). In Madagascar, hantavirus RNA was identified by molecular analysis

Author affiliations: Institut Pasteur de Madagascar, Antananarivo, Madagascar (H.A. Rabemananjara, V. Raharinosy,

R.M. Razafimahefa, J.P. Ravalohery, J.T. Rafisandratantsoa,

S.F. Andriamandimby, M. Rajerison, S. Rahelinirina, A. Harimanana, J. Irinantenaina, M.-M. Olive, C. Rogier, J.-M. Heraud, C. Filippone); Central Directorate of the French Military Health Service, Paris, France (C. Rogier); Institut Pasteur, Conakry, Guinea (N. Tordo); Institut Pasteur, Paris (N. Tordo, J.-M. Reynes, S. Petres); Federal Research Institute for Animal Health, Greifswald-Insel Riems, Germany (R.G. Ulrich); German Center for Infection Research, Hamburg-Luebeck-Borstel-Insel Riems, Germany (R.G. Ulrich); University of Aberdeen, Aberdeen, Scotland, UK (S. Telfer)

DOI: https://doi.org/10.3201/eid2603.190320 in Rattus rattus and Eliurus majori rats from a forest site in Anjozorobe district. The virus was named Anjozorobe virus (ANJZV) and is a genetic variant of Thailand orthohantavirus (THAIV) (5). In a more recent national study, Raharinosy et al. detected hantavirus RNA in $12 \%(\mathrm{n}=897)$ of $R$. rattus rats, and all the sequences obtained grouped with ANJZV (7), but they did not detect hantavirus RNA in $R$. norvegicus rats $(0 \% ; n=124)(7)$, a species commonly associated with the cosmopolitan Seoul orthohantavirus (1). Because THAIV may cause HFRS in Southeast Asia (8), ANJZV could also be a human pathogen in Madagascar. In 1986, a limited study that used an immunofluorescence assay with Hantaan orthohantavirus (HTNV) and Puumala orthohantavirus antigens was conducted in areas around the capital and reported low titer hantavirus antibodies in the serum samples of 7/18 rat catchers in Madagascar (9).

We conducted a national study to assess hantavirus exposure in the general population of Madagascar. Sampling took place in conjunction with a recent rodent survey (7). In addition, because the original molecular hantavirus detection in Madagascar was from forest rodents (5), we also collected and analyzed human and rat samples from 4 sites close to forests.

\section{The Study}

As part of a retrospective national study on zoonoses, we collected human serum samples from 20112013 . We then randomly recruited 1,680 asymptomatic participants (851 female and 829 male; average age 37 years; range 18-99 years). We conducted sampling in 28 sites, each with urban and rural zones;

${ }^{1}$ These first authors contributed equally to this article.

${ }^{2}$ These authors were co-principal investigators. 
Table 1. Seroprevalence of hantavirus in humans in the 28 sites used for national study, Madagascar*

\begin{tabular}{lcc}
\hline Site & & No. positive/total no. participants \\
no. & Site & $85 \%$ CI $)$ \\
\hline 1 & Antananarivo & $0 / 60(13.3 ; 6.3-25.1)$ \\
2 & Antsirabe & $1 / 60(1.7 ; 0.0-10.1)$ \\
3 & Anjozorobe & $3 / 60(5.0 ; 1.3-14.8)$ \\
4 & Tsiroanomandidy & $0 / 60(0.0 ; 0.0-7.5)$ \\
5 & Antsiranana & $2 / 60(3.3 ; 0.5-12.5)$ \\
6 & Sambava & $2 / 60(3.3 ; 0.5-12.5)$ \\
7 & Nosy-be & $1 / 60(1.7 ; 0.0-10.1)$ \\
8 & Mananjary & $3 / 60(5.0 ; 1.3-14.8)$ \\
9 & Ambositra & $4 / 60(6.7 ; 2.1-17.0)$ \\
10 & Farafangana & $0 / 60(0.0 ; 0.0-7.5)$ \\
11 & Ihosy & $1 / 60(1.7 ; 0.0-10.1)$ \\
12 & Fianarantsoa & $1 / 60(1.7 ; 0.0-10.1)$ \\
13 & Antsohihy & $0 / 60(0.0 ; 0.0-7.5)$ \\
14 & Mandritsara & $4 / 60(6.7 ; 2.1-17.0)$ \\
15 & Maevatanana & $0 / 60(0.0 ; 0.0-7.5)$ \\
16 & Ambato Boeny & $2 / 60(3.3 ; 0.5-12.5)$ \\
17 & Mahajanga & $0 / 60(0.0 ; 0.0-7.5)$ \\
18 & Moramanga & $2 / 60(3.3 ; 0.5-12.5)$ \\
19 & Toamasina & $0 / 60(0.0 ; 0.0-7.5)$ \\
20 & Ambatondrazaka & $1 / 60(1.7 ; 0.0-10.1)$ \\
21 & Miandrivazo & $2 / 60(3.3 ; 0.5-12.5)$ \\
22 & Ejeda & $1 / 60(1.7 ; 0.0-10.1)$ \\
23 & Morombe & $2 / 60(3.3 ; 0.5-12.5)$ \\
24 & Toliary & $2 / 60(3.3 ; 0.5-12.5)$ \\
25 & Taolagnaro & $2 / 60(3.3 ; 0.5-12.5)$ \\
26 & Ambovombe & $0 / 60(0.0 ; 0.0-7.5)$ \\
27 & Belo sur Tsiribihina & $2 / 60(3.3 ; 0.5-12.5)$ \\
28 & Morondava & $46 / 1,680(2.7 ; 2.0-3.7)$ \\
\hline Total & & \\
\hline${ }^{*}$ Results from IgG testing by commercial ELISA and custom ELISA \\
developed for Anjozorobe virus. & \\
\hline & & \\
& &
\end{tabular}

we sampled 60 persons per site, with 30 persons per zone (10). In addition, we used samples collected during 2015-2016 from 4 rural sites close to natural forest areas in Moramanga district, which is close to Anjozorobe district. For this study, we randomly selected 139 asymptomatic participants (31-36 persons per site; average age 29 years, range $5-75$ years). We also conducted trapping of the rat population in these 4 sites and randomly selected $237 R$. rattus rats (58-61 per site).

The national ethics committee of Madagascar authorized human studies (authorization no. 066-MSANP/CE on July 26, 2011; no. 049-MSANT/ CE on July 03, 2012). We conducted animal studies in accordance with Pasteur Institute animal use guidelines (https://www.pasteur.fr/en/file/2626/ download?token $=Y g O q 4 Q W 7)$. A committee of the Institut Pasteur de Madagascar approved the studies.

For the national study, we performed initial screening using the commercial Dobrava-Hantaan IgG EIA kit (Reagena Ltd, https://www.reagena. $\mathrm{com})$ based on the recombinant nucleocapsid (N) protein from HTNV. HTNV and THAIV, along with other Murinae-associated hantaviruses (Appendix Table 1, https://wwwnc.cdc.gov/EID/article/26/3/19-0320-
App1.pdf), exhibit close antigenic relationship (11). However, because 2-way cross-reactivity is not complete (12), we developed a new IgG ELISA based on ANJZV recombinant $\mathrm{N}$ protein produced by a baculovirus-mediated insect cell expression system. We used this assay to test all samples testing positive or borderline by the commercial kit and a subset of negative samples (Appendix). Based on the apparent increased detection ability of the ANJZV ELISA, we only used ANJZV ELISA for testing the human samples from the 4 sites close to forest areas.

After screening 1,680 serum samples with the commercial ELISA, we found 36 (2.1\%) positive and $26(1.5 \%)$ borderline samples. Using the custom ANJZV ELISA on these samples and a subset of 62 negative samples, we found 46 positive and 15 borderline (Appendix Tables 2, 3). Thus, the ELISA we developed specifically for ANJZV appeared to be more sensitive. To obtain a conservative estimate of seroprevalence, only samples testing positive by both assays or positive by 1 assay and borderline by the other were considered positive; testing yielded an overall prevalence of $2.7 \%(46 / 1,680 ; 95 \%$ CI $2.0 \%-$ $3.7 \%)$ in the population; 30 male $(1.8 \%)$ and 16 female $(0.9 \%)$ participants tested positive.

Seropositive participants came from 20 of the 28 study sites (0-13.3\% per site) distributed all over Madagascar (Table 1; Figure). Univariate generalized linear mixed models with site-zone as random effect indicated no effect of age, sex, or location (urban or rural), but we did find a slight suggestion of increased exposure in sites where our previous study (7) had detected infected rats (OR 3.0, 95\% CI $0.78-11.5 ; p=0.11$ ).

The Moramanga sites, situated close to forest, had significantly higher seroprevalence rates (7.2\%: $10 / 139 ; 95 \%$ CI $3.7 \%-13.2 \%$; range $3.2 \%-11.1 \%$ ) than the national study sites (Kruskal-Wallis test $\chi_{1}^{2}=4.65$; $p=0.03$ ) (Table 2; Figure). This finding may partly reflect the apparent higher sensitivity of the ANJZV ELISA used for the regional study. Because $2(n=62$, $3.2 \%$ ) national samples tested negative by the commercial ELISA were positive by ANJZV ELISA, and 1,558 national samples were not tested by ANJZV ELISA, the overall national seroprevalence could be $>2.7 \%(3.2 \% \times 1,558=50 ;(50+46) / 1,680=5.7 \%)$. Of interest, when we tested $R$. rattus rats from the 4 Moramanga sites by nested reverse transcription PCR using a protocol described previously (Appendix) (7), we also observed significantly higher infection rates than those for the national study sites; 77 of 237 rat samples were positive $(32.5 \%$; $95 \%$ CI $26.7 \%$ $38.9 \%$; range $19.0 \%-43.3 \%$; Kruskal-Wallis test 


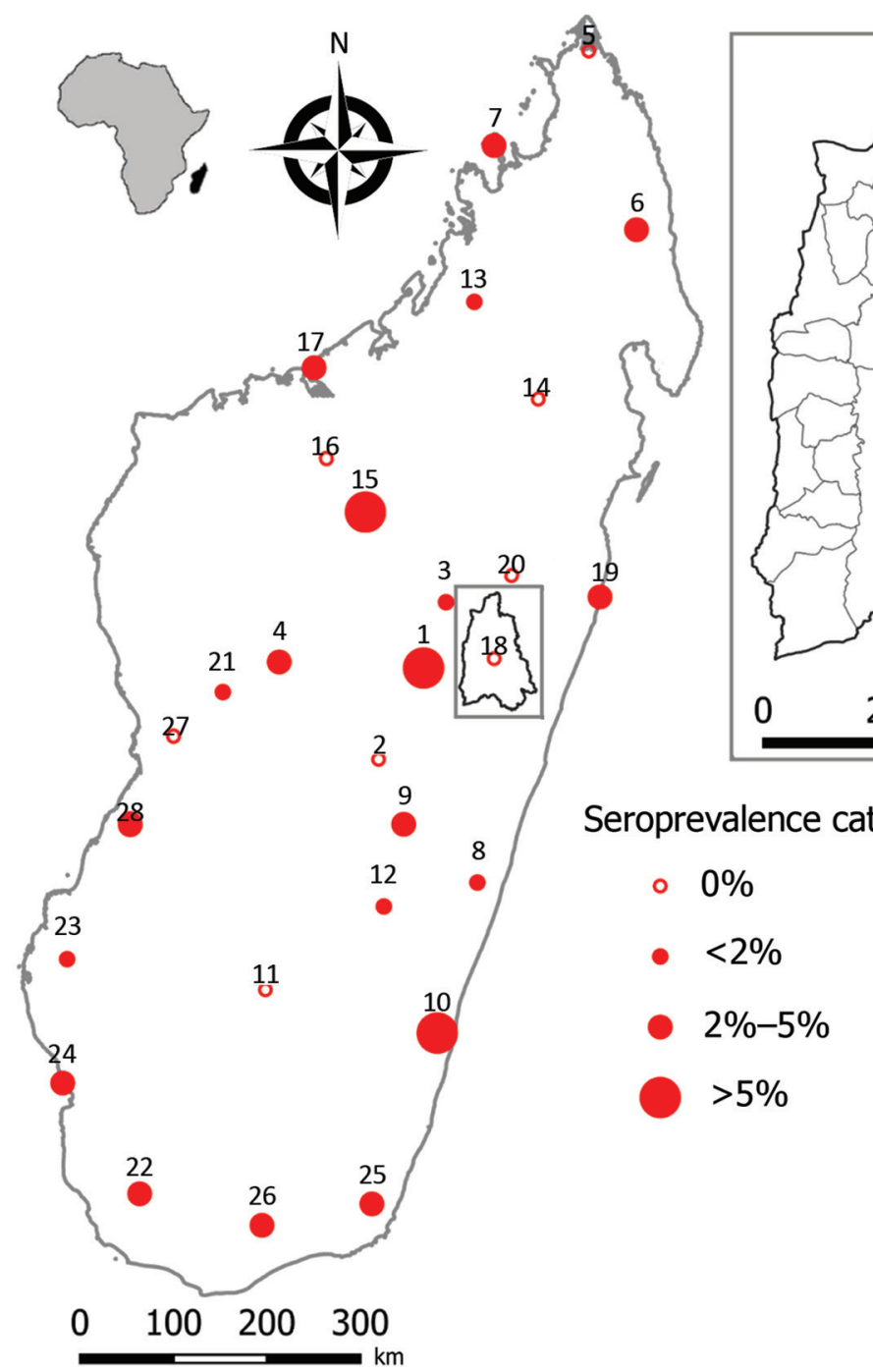

\begin{abstract}
Figure. Geographic distribution of IgG hantavirus human seroprevalence in Madagascar for the 28 sites of the national-scale study and (inset) for the 4 sites close to forest in Moramanga district. Maps were built with QGIS software version 3.8.0-Zanzibar (Open Source Geospatial Foundation Project, http://qgis.osgeo.org). Small inset map shows location of Madagascar off the coast of Africa. Details of results are provided in Tables 1 and 2.
\end{abstract}

$\left.X_{1}^{2}=5.55 ; p=0.02\right)$. These results further confirm a relatively high infection rate in the most abundant and widespread rodent in Madagascar. The small number of samples $(2 / 61 ; 3 \%)$ negative by ANJZV ELISA but seropositive by the commercial ELISA could be explained by other Murinae-associated hantaviruses circulating in Madagascar.

\section{Conclusions}

Our results suggest the population of Madagascar is exposed to hantaviruses associated with the Murinae subfamily of rodents. The overall conservative prevalence estimate of $2.7 \%$ from the national-scale study, obtained using 2 ELISA assays, is similar to results from studies in some Africa countries where other confirmatory tests were used $(3.9 \%$ in Cote d'Ivoire and $2.4 \%$ in the Democratic Republic of the Congo) $(4,13)$. Although we believe some seropositive persons may have been exposed to other Murinae-associated hantaviruses, considering both ELISA results in humans and rodent infection data together (7), our observations are consistent with evidence that most were exposed to ANJZV. Specifically, the ANJZV ELISA detected more seropositive persons than the commercial kit, and the cosmopolitan Seoul virus, if present in rodents in Madagascar, is at low prevalence or patchily

\begin{tabular}{|c|c|c|}
\hline $\begin{array}{l}\text { Site } \\
\text { no. }\end{array}$ & Site & $\begin{array}{c}\text { No. positive/total no. participants } \\
(\% ; 95 \% \mathrm{Cl})\end{array}$ \\
\hline I & Mangidifoza & $1 / 31(3.2 ; 0.1-18.5)$ \\
\hline II & Atsahatsaka & $4 / 36(11.1 ; 3.6-27.0)$ \\
\hline III & Sahamalotra & $3 / 36(8.3 ; 2.1-23.6)$ \\
\hline IV & Ambalafary & $2 / 36(5.5 ; 1.0-20.0)$ \\
\hline Total & & 10/139 (7.2; 3.7-13.2) \\
\hline
\end{tabular}


distributed (7). Because hantavirus infection rates in $R$. rattus rats appear higher at sites close to forest, more widespread testing with the ELISA developed for Anjozorobe virus is needed to confirm whether human communities in such areas are also at higher risk for infection. In addition, hospital surveillance studies are needed in Madagascar to determine if hantavirus infection occurs in patients, with testing focused on those with fever with unknown etiology, renal failure, or both.

\section{Acknowledgments}

We thank all colleagues from the Virology, Plague and Epidemiology Units of the Institut Pasteur de Madagascar who aided with field and laboratory work. We thank Corinne Jallet for help with some ELISA assays and Rasa Petraityte-Burneikiene and Aurelija Zvirbliene for providing antigen for additional analysis. We thank Reagena for constructive exchanges during provision of the kits.

This work was supported by Wellcome Trust Fellowships (no. 081705 and 095171 to S.T.) and Institut Pasteur de Madagascar, and by PTR (Progamme Transversal de Recherche) HANTAREV at Institut Pasteur, Paris.

\section{About the Author}

Mr. Rabemananjara is a PhD student at the Virology Unit of Institut Pasteur de Madagascar. His research interests include hantaviruses in animal reservoirs (bats and other small mammals) and humans, as well as the circulation of arboviruses in Madagascar.

\section{References}

1. Jonsson CB, Figueiredo LT, Vapalahti O. A global perspective on hantavirus ecology, epidemiology, and disease. Clin Microbiol Rev. 2010;23:412-41. https:/ / doi.org/ 10.1128/CMR.00062-09

2. Vaheri A, Strandin T, Hepojoki J, Sironen T, Henttonen H, Mäkelä S, et al. Uncovering the mysteries of hantavirus infections. Nat Rev Microbiol. 2013;11:539-50. https:// doi.org/10.1038/nrmicro3066

3. Muranyi W, Bahr U, Zeier M, van der Woude FJ. Hantavirus infection. J Am Soc Nephrol. 2005;16:3669-79. https://doi.org/10.1681/ASN.2005050561

4. Witkowski PT, Klempa B, Ithete NL, Auste B, Mfune JKE, Hoveka J, et al. Hantaviruses in Africa.
Virus Res. 2014;187:34-42. https:/ / doi.org/10.1016/ j.virusres.2013.12.039

5. Reynes JM, Razafindralambo NK, Lacoste V, Olive MM, Barivelo TA, Soarimalala V, et al. Anjozorobe hantavirus, a new genetic variant of Thailand virus detected in rodents from Madagascar. Vector Borne Zoonotic Dis. 2014;14:212-9. https://doi.org/10.1089/vbz.2013.1359

6. Filippone C, Castel G, Murri S, Beaulieux F, Ermonval M, Jallet $C$, et al. Discovery of hantavirus circulating among Rattus rattus in French Mayotte island, Indian Ocean. J Gen Virol. 2016;97:1060-5. https://doi.org/10.1099/jgv.0.000440

7. Raharinosy V, Olive MM, Andriamiarimanana FM, Andriamandimby SF, Ravalohery JP, Andriamamonjy S, et al. Geographical distribution and relative risk of Anjozorobe virus (Thailand orthohantavirus) infection in black rats (Rattus rattus) in Madagascar. Virol J. 2018;15:83. https:/ / doi.org/10.1186/s12985-018-0992-9

8. Pattamadilok S, Lee BH, Kumperasart S, Yoshimatsu K, Okumura M, Nakamura I, et al. Geographical distribution of hantaviruses in Thailand and potential human health significance of Thailand virus. Am J Trop Med Hyg. 2006;75:994-1002. https:/ / doi.org/10.4269/ajtmh.2006.75.994

9. Rollin PE, Mathiot C, Nawrocka E, Ravaoalimalala VE, Coulanges P, Sureau P, et al. Hemorrhagic fever with renal syndrome in Madagascar. First seroepidemiologic survey of rat populations [in French]. Arch Inst Pasteur Madagascar. 1986;52:181-6.

10. Olive MM, Chevalier V, Grosbois V, Tran A, Andriamandimby SF, Durand B, et al. Integrated analysis of environment, cattle, and human serological data: risk and mechanisms of transmission of Rift Valley fever in Madagascar. PLoS Negl Trop Dis. 2016;10:e0004827. https://doi.org/10.1371/journal.pntd.0004827

11. Yoshimatsu K, Arikawa J. Serological diagnosis with recombinant $\mathrm{N}$ antigen for hantavirus infection. Virus Res. 2014; 187:77-83. https:/ / doi.org/10.1016/j.virusres.2013.12.040

12. Chu YK, Rossi C, Leduc JW, Lee HW, Schmaljohn CS, Dalrymple JM. Serological relationships among viruses in the Hantavirus genus, family Bunyaviridae. Virology. 1994;198:196-204. https://doi.org/10.1006/viro.1994.1022

13. Witkowski PT, Leendertz SAJ, Auste B, Akoua-Koffi C, Schubert G, Klempa B, et al. Human seroprevalence indicating hantavirus infections in tropical rainforests of Côte d'Ivoire and Democratic Republic of Congo. Front Microbiol. 2015;6:518. https:/ / doi.org/10.3389/ fmicb.2015.00518

Address for correspondence: Claudia Filippone, Institut Pasteur de Madagascar-Virology Unit, Ambatofotsikely, BP 1274

Antananarivo, Madagascar; email: cla.filippone@gmail.com; Sandra Telfer, University of Aberdeen, Institute of Biological and Environmental Sciences, Aberdeen, Scotland AB24 2TZ, UK; email: s.telfer@abdn.ac.uk 